\title{
Bigeneric Nomina: An Historical and Evolutionary Perspective
}

\author{
ROBERT B. ECKHARDT \\ Laboratory of Physical Anthropology, Departments of Anthropology and \\ Human Genetics, The University of Michigan, Ann Arbor, Michigan 48104
}

\begin{abstract}
It is suggested that the addition of bigeneric or bispecific nomina to designate fossils which are intermediate between two chronogenera or chronospecies is inadvisable, because the use of either of these categories to describe cases involving a single phyletic line, consisting of a continuous chain of time-sequential populations, misrepresents the nature of the evolutionary processes involved; and that in such cases fossils might best be referred to by specimen numbers.
\end{abstract}

In re-reading Raymond Dart's epochmaking paper "Australopithecus africanus: The Man-Ape of South Africa" ('25), this writer was reminded of Tobias's recent ('69) proposal for the modification of the International Rules of Zoological Nomenclature to allow the use of bigeneric nomina to designate a group of fossils which are intermediate between two consecutive genera, and bispecific nomina to designate fossils which are intermediate between two consecutive species.

In his evaluation of the significance of the juvenile Taungs skull designated as Australopithecus africanus, Dart noted that it represented

. . a creature well advanced beyond modern anthropoids in just those characters, facial and cerebral, which are to be anticipated in an ex. tinct link between man and his simian ancestor. At the same time, it is equally evident that a creature with anthropoid brain capacity and lacking the distinctive, localised temporal expansions which appear to be concomitant with and necessary to articulate man, is no true man. It is therefore logically regarded as a man-like ape. I propose tentatively, then, that a new family of Homo-simiadae ${ }^{1}$ be created for the reception of the group of individuals which it represents. . .

Dart's proposal with regard to taxonomic categories at the family level is paralleled by that of Tobias at the levels of genera and species; as both indicate a recognition of the continuity of hominid descent, and an understanding of the evolutionary processes involved, they have much to recommend them. Nonetheless, this does not in itself establish a sufficient cause for the adoption of systems of bigeneric or bi- specific nomina. It is evident that fossil hominids are animals and, as Simpson ('63) has emphasized, the appropriate language for discussing their classification and relationships is that of animal taxonomy. The principles governing this language are contained in the Rules of Zoological Nomenclature (Stoll et al., '61), which do not at present sanction dual systems of nomenclature.

Tobias's suggestion was prompted by the group of fossils consisting of Olduvai Bed I hominids 4, 6, 7, and 8, and Olduvai Bed II hominids 13,14 , and perhaps 16 . Tobias noted that in addition to the original idea that these remains belong to a separate taxon, Homo habilis, at least five additional taxonomic names had already been applied to them by different workers, although most are agreed that the habilis group of fossils represents "an intermediate form" between Australopithecus and Homo.

Part of the disagreement in this case, as recognized by Tobias, arises from the necessity of applying names which indicate discontinuous categories to specimens which represent samples drawn from a continuum of hominid populations which followed one another sequentially through time. But the use of bigeneric or bispecific nomina will not, in the long run, provide a satisfactory solution to this problem.

If one accepts "Homo habilis" as a form intermediate between Australopithecus

1 As is generally quite well known, this nomen is technically incorrect on several grounds: family names must be based on generic names, must not be hyphenated, and must end in -idae. 
africanus and Homo erectus and shows this by making it

Australopithecus

Homo

habilis

or its more easily printed equivalent, Australopithecus/Homo habilis (Ewer, '67), what does one do with the forms - which must have existed and probably will be found - between, for example, Australopithecus /Homo habilis and Homo erectus? If it is generally acknowledged that with these hominids we are dealing with an evolutionary continuum, the use of a series of names such as

\section{Australopithecus africanus Australopithecus/Homo habilis and Homo erectus}

simply replaces two taxonomic categories, $A$. africanus and $H$. erectus, with the three above, without increasing our understanding of the evolutionary processes involved; and it is doubtful that those who do not accept the idea that an evolutionary series is represented here will be induced to accept the name applied to the intermediate category.

In his article Tobias asked "Granted that bigger samples confirm that Homo habilis is an intermediate form, how are we to name such formes de passage?" The answer to this question depends on the purpose for which such names are sought. Taxonomic nomina are not meant to be applied as labels for one or a few specimens. For purposes of discussion of individual specimens, use of their specimen numbers: Olduvai I hominids $4,6,7,8$, etc., should be quite sufficient. Simpson ('63) has noted that a designation such as this - by a collection or repository symbol, and a catalogue number referring uniquely to each specimen - is practically universal in zoology, although not yet in anthropology. ${ }^{2}$ If this procedure were adopted it might have, aside from simplicity, at least one other salient advantage to recommend it: immediate descriptions of such material could be published (hopefully accompanied by standardized measurements and photographs or line drawings, and ideally followed by the production of accurate casts for study by others) without the necessity either for making a hasty judgment about the taxonomic status of the specimen, or the alternative of delaying publication of a description until such a decision about the proper taxonomic position has been lengthily considered. This would enable other workers in the field to come to their own conclusions on the subject, and might either eliminate much of the debate which often accompanies such taxonomic designations, or at least provide a more substantive basis for the ensuing discussions

The practice suggested here, of referring to fossils by their specimen numbers, does not preclude the assignment of proper taxonomic names to given specimens, nor does it suggest any criteria which might be used; it does obviate the necessity for drawing numerous fine taxonomic distinctions if doing so, in itself, adds nothing to our understanding of the evolutionary process. There is a strong implication here that where the case is one of phyletic evolution, and forms representing a number of intermediate populations are known, perhaps a simple taxonomic system, intelligently used, is the best. Such a system was suggested by Mayr ('50): that fossil and recent hominids be classified into a single genus (Homo) with three time-successive species (transvaalensis, ${ }^{3}$ erectus, and sapiens). Although Mayr subsequently altered his opinion on this to accept the more standard view that in South Africa during the Upper Villafranchian there were two distinct contemporaneous types of early hominids, Australopithecus and Paranthropus, which merit generic separation from each other and from Homo, some other students of human evolution, this writer included, still believe that the evidence favors his earlier position.

Uniting all of the above hominids into the simple genus Homo is done in recognition of the ecological significance of upright, bipedal posture and tool use, which one can consider to have placed all hominids, even the earliest ones, onto a unique

\footnotetext{
2 The problems which arise because of the failure to follow this practice are not confined to olduvai to follow this practice are nother example, no one maintains that Telanthropus is a valid taxon at the generic level, but no way has been found to refer to the specimens in question except as Telanthropus, an $\mathrm{N}_{3}$ designation [the technical Neo-Linnaean name, or nomen] that necessarily implies a taxonomic conclusion agreed to be incorrect" (Simpson, '63)

3 Thirect" (Simpson, '63). should be Homo africanus rather than Homo transvaalensis.
} 
adaptive plateau (others might consider the adaptive zone or plateau of hominids to be defined by different characteristics, and on these grounds exclude the earlier forms from the genus Homo). It might thus be maintained that all of the abovementioned hominids should be considered as an evolutionary species; according to Simpson ('61) this is "a lineage (an ancestral-descendant sequence of populations) evolving separately from others and with its own unitary evolutionary role and tendencies." Any subdivision of this lineage into successive species as done by Mayr ('50) would of course be arbitrary, but nevertheless consistent with standard paleontological procedure if the morphological differences between the successive species were as great as those separating contemporaneous species of one genus. In any case classifications cannot be expected to fully express all that is known about the genetic and evolutionary relationships of the organisms concerned (Simpson, '62).

By the application to hominid taxonomy of these procedures common to other branches of animal taxonomy, the number of arbitrary subdivisions of a lineage would be kept to a minimum, contrasting with Tobias's proposal for the creation of bigeneric and bispecific nomina, which would result in overly fine subdivisions of an evolutionary continuum, a clumsier nomenclature, and the creation of an illusion of nonexistent taxonomic complexity.

\section{LITERATURE CITED}

Dart, R. A. 1925 Australopithecus africanus: The Man-Ape of South Africa. Nature, 115: 195-199.

Ewer, R. F. 1967 Professor Tobias's new nomenclature. S. Afr. J. Sci., 63: 281.

Mayr, E. 1950 Taxonomic Categories in Fossil Hominids. Cold Spring Harbor Symposia on Quantitative Biology, 15; 109-118.

1963 Animal Species and Evolution. Belknap Press, Cambridge.

Simpson, G. G. 1961 Principals of Animal Taxonomy. Columbia Univ. Press, New York. 1962 Primate Taxonomy and recent studies of nonhuman primates. In: The Relatives of Man: Modern Studies of the Relation of the Evolution of Nonhuman Primates to Human Evolution. J. Buettner-Janusch, ed. Ann. N. Y. Acad. Sci., 102: 497-514.

1963 The meaning of taxonomic statements. In: Classification and Human Evolution. S. L. Washburn, ed. (Viking Fund Publication in Anthropology, No. 37) Aldine, Chicago.

Stoll, N. R., R. Ph. Dollfus, J. Forest, N. D. Riley, C. W. Sabrosky, C. W. Wright, R. V. Melville, eds. 1961 International Code of Zoological Nomenclature. International Trust for Zoological Nomenclature, London (rev, ed. London, 1964).

Tobias, P. V. 1969 Bigeneric Nomina: A Proposal for Modification of the rules of nomenclature. Am. J. Phys. Anthrop., 31: 103-105. 